\title{
Effects of Culture Conditions on a Micropatterned Co-culture of Rat Hepatocytes with 3T3 cells
}

\author{
Kohji Nakazawa*, Yukako Shinmura, Ami Higuchi and Yusuke Sakai
}

Department of Life and Environment Engineering, The University of Kitakyushu 1-1 Hibikino, Wakamatsu-ku, Kitakyushu, Fukuoka 808-0135, Japan

\begin{abstract}
We investigated the effect of culture conditions on the micropatterned co-culture of rat hepatocytes with $3 T 3$ cells. A micropatterned chip was prepared using polydimethylsiloxane (PDMS) microstencil such that the chip contained 724 hepatocyte islands, each $500 \mu \mathrm{m}$ in diameter, in a triangular arrangement with $800-\mu \mathrm{m}$ pitch, in which hepatocytes were co-cultured with $3 T 3$ cells. The hepatocytes in micropatterned co-culture exhibited hepatocellular morphology, and the micropatterned configuration of hepatocyte islands was maintained for several weeks of culture by supporting the heterotypic interface between the hepatocytes and 3T3 cells. The albumin secretion activity of hepatocytes was highest in the micropatterned co-culture but decreased in the random co-culture, micropatterned mono-culture (hepatocytes only), and random mono-culture (hepatocytes only) in that order. Furthermore, earlier formation of co-culture promoted higher functional activity of hepatocytes as compared to later formation, and hepatocyte functions were induced with an increasing the density of inoculated 3T3 cells. These results suggest that the formation of a heterotypic interaction at an early stage is important for maintaining high levels of hepatocyte functions. The findings of this study will provide information useful for designing co-culture conditions for liver tissue engineering and pharmacological and toxicological studies.
\end{abstract}

Keywords: Micropatterned culture; Co-culture, Microstencil; Rat hepatocytes; $3 \mathrm{~T} 3$ cells; Albumin secretion

\section{Introduction}

The liver plays many essential roles in maintaining normal physiology. Therefore, primary hepatocytes have been used for various applications such as liver tissue engineering and pharmacological, toxicological, and fundamental cell biology studies. For the success of such applications, hepatocytes have to express liver-specific functions at a high level and maintain these functions over a long term. Expression of liver-specific functions in the hepatocytes is closely related to the in vitro cell configuration and culture environments. Various approaches have been adopted to preserve hepatocyte functions in vitro. These include optimization of medium components [1-3], studies of extracellular matrices [4-6], construction of spheroid culture [7-9], and adoption of co-culture techniques. Co-culturing hepatocytes with non-parenchymal cells or fibroblasts is one approach that is useful for regulating culture environments because it is known that hepatocytes can maintain cell viability and liver-specific functions such as albumin synthesis, urea production, and detoxification, including cytochrome P450 activities, in long-term culture [10-15].

Although many previous studies on co-culture have used random culture in which cell distribution is heterogeneous, micropatterned co-cultures have been established in recent studies [16-25]. Micropatterned cultures have the following advantages over random cultures: they can control cellular microenvironments by regulating cell arrangement on a micro-scale. Furthermore, micropatterned co-cultures of hepatocytes with non-parenchymal cells or fibroblasts have provided beneficial information regarding heterotypic cell-cell communication, demonstrating that the expression of hepatocyte functions is stabilized by the increase of heterotypic cell-cell contacts between the hepatocytes and other cell types [19-22,25].

Among all techniques for micropatterned co-culture, a microstencil method is a simple yet effective technique: the cell pattern is easily formed on the culture plate by peeling off a microstencil, which is a thin membrane with orderly through-microholes [23-25]. Furthermore, this method can also be used to evaluate the relationship between coculture conditions and functional behaviors of cells. Determining such a relationship may require establishing the optimum conditions for micropatterned co-culture.

In this study, we focused on the differences of culture conditions on the micropatterned co-culture of rat hepatocytes with 3T3 cells, and the morphological and functional behaviors of the micropatterned co-culture compared to a random co-culture. Furthermore, the effects of co-culture timing and inoculated density of 3T3 cells on the expression of hepatocyte functions in the micropatterned co-culture were evaluated. This study aimed to demonstrate the advantages of micropatterned co-culture compared to random co-culture and to specify the optimum conditions for the micropatterned co-culture.

\section{Materials and Methods}

\section{Preparation of a microstencil chip}

Figure 1A shows a schematic diagram of the microstencil chip. A polydimethylsiloxane (PDMS; Sylgard 184, Dow Corning Co., Midland, MI, USA) microstencil, which consists of a thin membrane with 724 through-microholes, each $500 \mu \mathrm{m}$ in diameter, in a triangular arrangement of $800-\mu \mathrm{m}$ pitch, was fabricated by peeling the microstencil off from a microfabricated polymethylmethacrylate (PMMA) mould. The limitation of microstencil diameter that could make it by this

*Corresponding author: Kohji Nakazawa, Department of Life and Environment Engineering, The University of Kitakyushu,1-1 Hibikino, Wakamatsu-ku, Kitakyushu, Fukuoka 808-0135, Japan,Tel: +81-93-695-3292,Fax: +81-93-6953359; E-mail: nakazawa@kitakyu-u.ac.jp

Received August 28, 2011; Accepted September 28, 2011; Published September 30, 2011

Citation: Nakazawa K, Shinmura Y, Higuchi A, Sakai Y (2011) Effects of Culture Conditions on a Micropatterned Co-culture of Rat Hepatocytes with 3T3 cells. J Bioprocess Biotechniq S3:002 doi:10.4172/2155-9821.S3-002

Copyright: (c) 2010 Nakazawa K, et al. This is an open-access article distributed under the terms of the Creative Commons Attribution License, which permits unrestricted use, distribution, and reproduction in any medium, provided the original author and source are credited. 
method was $500 \mu \mathrm{m}$. Furthermore, previous studies including us have reported that the micropatterned culture with hepatocyte islands of 500 $\mu \mathrm{m}$ in diameter maintains high expression of liver-specific functions as compared to the conventional monolayer culture $[20,25,26]$. Therefore, we adopted this microstencil condition. To prepare the microstencil chip, the fabricated microstencil was sealed onto the surface of a glass plate $(24 \times 24 \mathrm{~mm})$, and the surface of the microstencil chip was coated with type IV collagen to promote cell adhesion Figure 1B. The chip was sterilized by immersion in $70 \%$ ethanol, thoroughly rinsed with distilled water, and immersed in the culture medium until use.

\section{Micropatterned culture of rat hepatocytes}

This experiment was reviewed by the Committee of Ethics on Animal Experiments of our institute and conducted as per the Guidelines for Animal Experiments at our institute.

Hepatocytes were isolated from the whole liver of an adult Wistar rat (male, 7-8 weeks old, and weighing approximately $200 \mathrm{~g}$ ) by perfusion with $0.05 \%$ collagenase (Wako Pure Chemical Industries Ltd., Osaka, Japan) [27]. Cell viability was determined by the trypan blue exclusion method, and cells that exhibited $>85 \%$ viability were used for subsequent experiments.

The culture medium was Dulbecco's modified Eagle's medium (DMEM; Invitrogen Corp., Carlsbad, CA, USA) supplemented with $10 \%$ fetal bovine serum (FBS), $10 \mathrm{mg} / \mathrm{L}$ insulin (Sigma, St. Louis, MO, USA), $7.5 \mathrm{mg} / \mathrm{L}$ hydrocortisone (Wako), $50 \mu \mathrm{g} / \mathrm{L}$ epidermal growth factor (Biomedical Technologies Inc., Stoughton, MA, USA), $60 \mathrm{mg} / \mathrm{L}$ proline (Wako), $50 \mu \mathrm{g} / \mathrm{L}$ linoleic acid (Sigma), $0.1 \mu \mathrm{M} \mathrm{CuSO}_{4} \cdot 5 \mathrm{H}_{2} \mathrm{O}, 3$ $\mu \mathrm{g} / \mathrm{L} \mathrm{H}_{2} \mathrm{SeO}_{3}, 50 \mathrm{pM} \mathrm{ZnSO} \cdot 7 \mathrm{H}_{2} \mathrm{O}, 58.8 \mathrm{mg} / \mathrm{L}$ penicillin, and $100 \mathrm{mg} / \mathrm{L}$ streptomycin.

Figure 1B shows the process of creating micropatterned culture of hepatocytes using the microstencil chip. Hepatocytes $\left(1.0 \times 10^{6}\right)$ were inoculated onto the microstencil chip that was placed in a polystyrene dish (diameter, $35 \mathrm{~mm}$ ) containing $2 \mathrm{~mL}$ of culture medium. After 24 $\mathrm{h}$, the stencil was peeled off the chip, and the chip with micropatterned hepatocytes was transferred to another polystyrene dish containing 2 $\mathrm{mL}$ of fresh culture medium (micropatterned mono-culture). By this
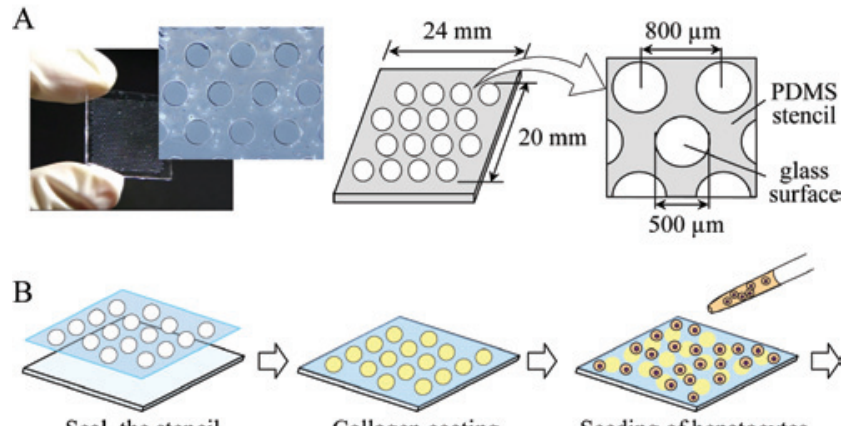

Seal the stencil
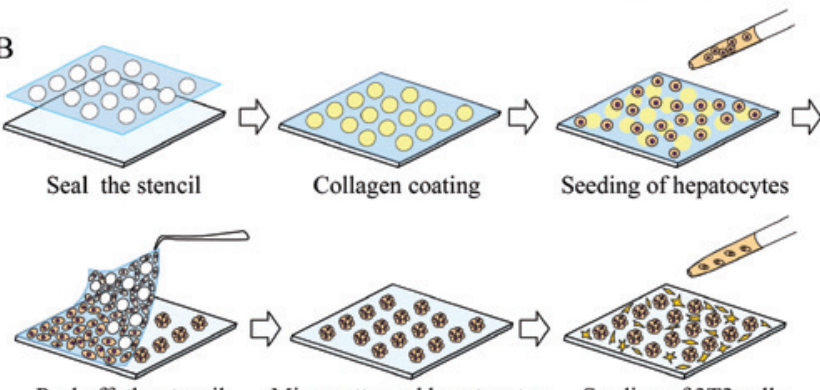

Peel off the stencil
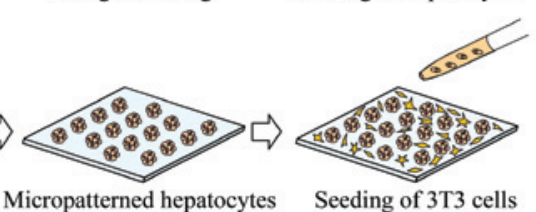

Figure 1: (A) Schematic diagram of the microstencil chip. (B) Process of micropatterned co-culture of rat hepatocytes with 3 T3 cells using the microstencil chip. procedure, approximately $7.0 \times 10^{4}$ cells were immobilized on the chip after $24 \mathrm{~h}$ of culture. To obtain a random mono-culture, hepatocytes $\left(2.5 \times 10^{5}\right)$ were inoculated onto a $35-\mathrm{mm}$ dish coated with type IV collagen. Approximately $9.0 \times 10^{4}$ cells were immobilized on the dish after $24 \mathrm{~h}$ of culture.

\section{Micropatterned co-culture of rat hepatocytes with 3T3 cells}

NIH/3T3 cells (JCRB0615; Health Science Research Resources Bank, Osaka, Japan) were subcultured as a continuous monolayer in a 100-mm tissue culture dish (Corning, Corning, NY, USA) containing $10 \mathrm{~mL}$ DMEM supplemented with $10 \%$ FBS, $100 \mathrm{U} / \mathrm{mL}$ penicillin, and $100 \mu \mathrm{g} / \mathrm{mL}$ streptomycin. For co-culture experiments, the 3T3 cells were dispersed by treating the confluent monolayer formed on the tissue culture dish with $0.25 \%$ trypsin (Invitrogen), and the cells were suspended in the same medium with rat hepatocyte culture.

To compare the effect between micropatterned co-culture and random co-culture, $5.0 \times 10^{5} 3 \mathrm{~T} 3$ cells were inoculated onto the micropatterned- and random-hepatocyte cultures at day 1 . To evaluate the effect of co-culture timing, $5.0 \times 10^{5} 3 \mathrm{~T} 3$ cells were inoculated onto the micropatterned hepatocyte culture at days 1,3 , and 5 of culture. To evaluate the effect of inoculated density of $3 \mathrm{~T} 3$ cells, cell suspensions of densities $1.0 \times 10^{5}$ and $5.0 \times 10^{5}$ cells were inoculated onto the micropatterned hepatocyte cultures at 3 days of culture. The culture medium was changed at 2-day intervals. All cells were cultured at $37^{\circ} \mathrm{C}$ in a humidified atmosphere of $5 \% \mathrm{CO}_{2}$ and $95 \%$ air.

\section{Albumin secretion activity}

Albumin secretion activity was evaluated as a typical liver-specific function. The concentrations of albumin secreted into the culture medium during the $24 \mathrm{~h}$ of culture were determined by performing an enzyme-linked immunosorbent assay (ELISA). We used twoantibody sandwich method. Briefly, goat anti-rat albumin antibody (MP Biomedicals, Capple Products, USA) was bound to each well of a 96-well microtiter plate, and then the samples were applied to the wells. Subsequently, peroxidase-conjugated sheep anti-rat albumin antibody (MP Biomedicals) was added to each well. ABTS (KPL; Kirkegaard \& Perry Laboratories, Inc., USA) was used as a chromogenic substrate, and a microplate reader (Model 550, Bio-Rad Laboratories, USA) was used for the measurement. The albumin concentration of each sample was calculated from the standard curve of rat albumin (MP Biomedicals). The activity was evaluated on days 3, 5, 10, 15, and 20 of the culture, and the values were normalized with the immobilized cells at day 1 of culture.

\section{Statistical analysis}

Data obtained from the albumin secretion of rat hepatocytes are represented as mean \pm SD of 3 points. Statistical analysis of the numerical variables was performed using a repeated-measures ANOVA test. A p value of $<0.05$ was considered significant.

\section{Results and Discussion}

\section{Cell morphology}

In the random mono-culture, rat hepatocytes adhered to the collagen-coated plate and formed a monolayer; however, cell distribution was heterogeneous throughout during the culture Figure $2 \mathrm{~A}$. In the random co-culture, the inoculated $3 \mathrm{~T} 3$ cells spread onto the interstitial space between the adhered hepatocytes, and the hepatocytes exhibited heterogeneous colony-like morphology by the proliferation 
of $3 \mathrm{~T} 3$ cells Figure 2B. Although hepatocytes in the random monoculture rapidly lost cuboidal morphology by elongation of cells, they maintained bright intercellular borders with distinct nuclei after longterm culture in the random co-culture, indicating that the co-culture is effective in maintaining the hepatocellular morphology.

Although the hepatocytes inoculated onto the microstencil chip adhered to the surface of the microstencil and to the glass surface in through-microholes of the microstencil and formed a monolayer within 1 day of culture, the micropatterned hepatocyte islands were formed by peeling off the stencil Figure 2C. The configuration of micropatterned hepatocytes was maintained until 5 days of culture; however, the hepatocytes were elongated at the chip surface after it was removed by the stencil. Consequently, the circular pattern of hepatocytes gradually decayed after 5-7 days of culture Figure 2E. In contrast, in the micropatterned co-culture, the 3T3 cells proliferated in the gap between the hepatocyte islands, thus forming the clear heterotypic interface between the hepatocytes and 3T3 cells Figure 2D. The hepatocytes exhibited hepatocellular morphology, and the configuration of micropatterned hepatocyte islands was also maintained for several weeks of culture by supporting the heterotypic interface Figure $2 \mathrm{~F}$. The result that the co-culture maintained the hepatocellular morphology irrespective of the random or micropatterned culture corresponds well with those of previous studies $[19,20,25]$, probably because hepatocyte elongation is inhibited when the substratum surface is covered by $3 \mathrm{~T} 3$ cells.

\section{Effect of the micropatterned co-culture}

Among the various liver-specific functions, we chose albumin secretion activity as an index for the effect of co-culture because albumin secretion is the hepatocyte function that is known to be elevated by co-culture [10-25].

Figure 3 shows changes in the albumin secretion activities of hepatocytes under 4 culture conditions: random mono-culture, random co-culture, micropatterned mono-culture, and micropatterned co-culture. The albumin secretion activity was maintained at a higher level in co-cultures than in mono-cultures, irrespective of whether the culture was random or micropatterned. Furthermore, the secretion activity was maintained at a higher level in micropatterned co-culture than in random co-culture, indicating that micropatterned co-culture is most useful method of maintaining liver-specific functions. Our previous study of mono-culture (with only hepatocytes) revealed
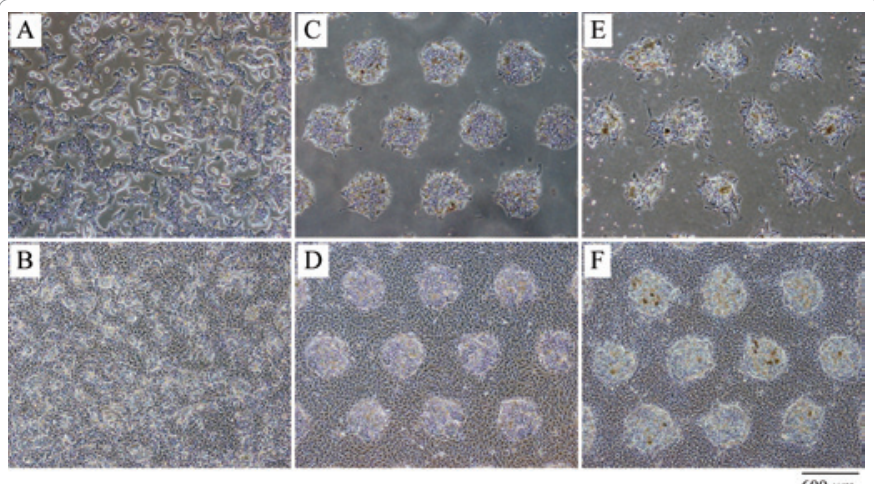

Figure 2: Phase-contrast micrographs of different culture conditions. (A) Random mono-cultures; $(\mathrm{B})$ random co-culture; ( $\mathrm{C}$ and $\mathrm{E}$ ) micropatterned monoculture; and (D and F) micropatterned co-culture. A-D, day 3 of culture; E, day 7 of culture; and $F$, day 15 of culture.

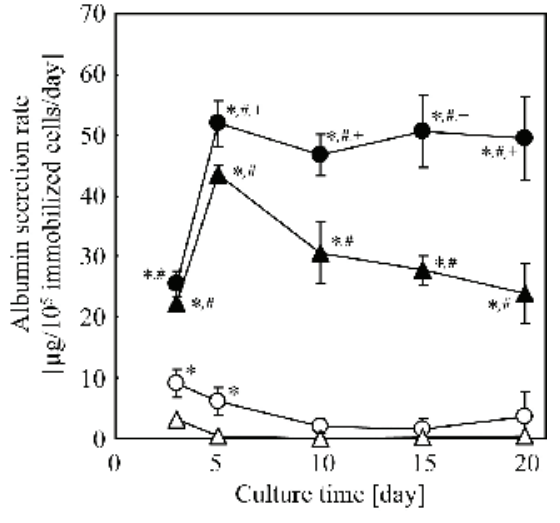

Figure 3: Changes in albumin secretion activity of hepatocytes on random mono-culture (open triangles), random co-culture (closed triangles), micropatterned mono-culture (open circles), and micropatterned co-culture (closed circles). Error bars represent SD; ${ }^{*}, p<0.05$ compared with the value of random mono-culture; \#, $p<0.05$ compared with the value of micropatterned monoculture; and ${ }^{+}, p<0.05$ compared with the value of random co-culture.

that hepatocytes in a micropatterned culture maintained normal hepatocellular morphology and stable homotypic cell-cell contacts (cell adhesion and gap junction in the hepatocytes); consequently, the expression of liver-specific functions was well retained as compared with the random monolayer [26]. This difference may be reflected to the difference of function between the random and micropatterned co-cultures. Although the maintenance of high functional expression in the mono-culture was difficult even if it was the micropatterned culture, the functional expression was drastically improved by the co-culture. Thus, hepatocytes in the micropatterned co-culture may be able to develop higher functions due to the synergistic effects of improving the culture environment by co-culture and stabilizing the hepatocyte functions by micropatterned culture.

\section{Effect of the co-culture conditions}

To evaluate the effect of the micropatterned co-culture conditions, the albumin secretion activities of hepatocytes were compared before and after changing the co-culture timing and inoculation density of 3T3 cells.

The albumin secretion activity of hepatocytes in the micropatterned co-culture decreased on day 3 as compared to day 1 and on day 5 as compared to day 3 Figure 4 . Thus, earlier formation of co-culture induces higher functional activities than does later formation. Although recovery of hepatocyte functions by co-culture has been reported [10], to the best of our knowledge, this is the first study that reports that the expression of hepatocyte functions differs depending on the co-culture timing. This finding shows that early formation of co-culture is necessary to maintain a high functional expression of hepatocytes. The activity of micropatterned mono-culture (with only hepatocytes) gradually decreases with the increase in the culture time. This phenomenon may occur by the lack of stimulations such as cell signaling factors, extracellular matrixes, and cell-cell contacts from other cells. Therefore, earlier formation of co-culture may shorten those lack periods, and consequently, the expression of hepatocyte functions may be well maintained.

Furthermore, the albumin secretion activity of hepatocytes in the micropatterned co-culture inoculated at a density of $5 \times 10^{5} 3 \mathrm{~T} 3$ cells was higher than that in a co-culture inoculated at a density of $1 \times 10^{5}$ 
$3 \mathrm{~T} 3$ cells, indicating that forming a co-culture with a high density of $3 \mathrm{~T} 3$ cells at the initial stage of the culture is superior for maintaining the high functional expression of hepatocytes Figure 5. Bhatia et al. reported that hepatocyte functions in random co-culture were induced depending on an increase of inoculated 3T3 cell density [19]; similarly, we have showed that the same phenomena exist in the micropatterned co-culture.

It is known that co-culture improves culture environment, since it induces production of cell signaling factors and extracellular matrixes, and/or formation of heterotypic cell-cell interaction. In particular, recent studies of co-culturing hepatocytes with $3 \mathrm{~T} 3$ cells revealed that the expression of hepatocyte functions is stabilized by an increase of direct heterotypic cell-cell contacts (cell adhesion and gap junction) [19-22,25,28]. This information corresponds with our results that the earliest formation of co-culture under high cell density of 3T3 cells induced the highest functional activities of hepatocytes. The direct heterotypic cell-cell contacts are important factors for hepatocellular

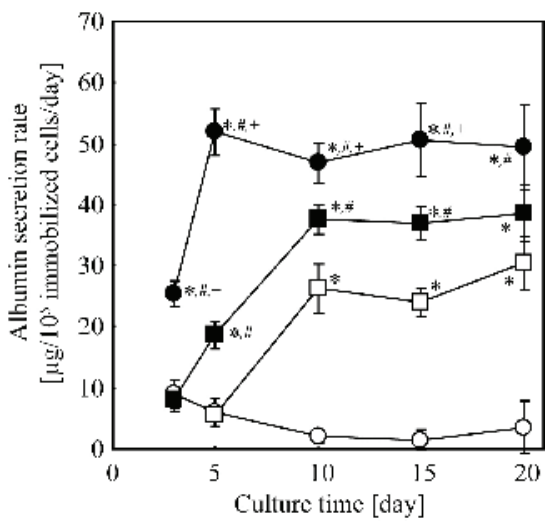

Figure 4: Effect of the co-culture timing on the albumin secretion activity of hepatocytes on micropatterned mono-culture (open circles), micropatterned co-culture inoculated on day 1 (closed circles), micropatterned co-culture inoculated on day 3 (closed squares), and micropatterned co-culture inoculated on day 5 (open squares) of culture. Error bars represent SD. ', $p<0.05$, compared with the value of mono-culture. ${ }^{\#}, p<0.05$, compared with the value of co-culture at day $5 .^{+}, p<0.05$, compared with the value of co-culture at day 3 .

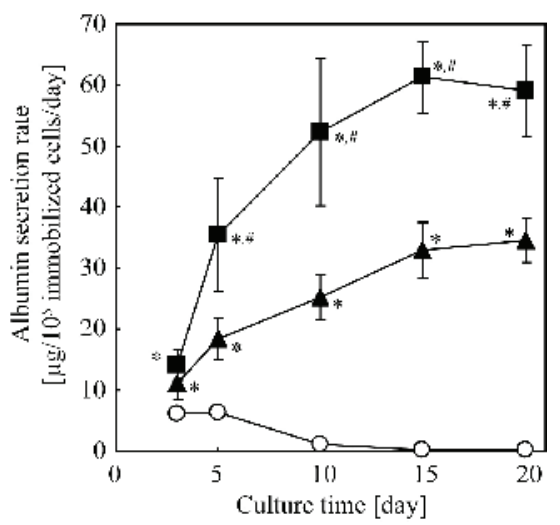

Figure 5: Effect of the inoculated density of $3 T 3$ cells on albumin secretion activity of hepatocytes. Micropatterned mono-culture (open circles); micropatterned co-culture inoculated with a 3 T 3 cell density of $1 \times 10^{5}$ cells (closed triangles); and micropatterned co-culture inoculated with a 3T3 cell density of $5 \times 10^{5}$ cells (closed squares). Error bars represent SD; ', $p<0.05$ compared with the value of the mono-culture;,$p<0.05$ compared with the value of the co-culture at $1 \times 10^{5} 3$ T3 cells. polarity and organization $[21,25]$. Although the detailed mechanism is not clear, their development may operate to normal hepatocellular structure that includes cuboidal cell shape and abundant cytoplasmic organelles; consequently, the expression of hepatocyte functions may be well maintained. Although the sufficient heterotypic cell-cell contacts are formed even if it was the random co-culture, the function of micropatterned co-culture was higher than that of random monolayer. This fact suggests that the combination of the heterotypic contacts (hepatocyte-3T3 cell) and the homotypic interactions (hepatocytehepatocyte) is important for maintaining the liver-specific functions. Further studies of heterotypic and homotypic interactions may help to better understand the mechanism.

\section{Conclusions}

In this study, we demonstrated the effect of culture conditions on the micropatterned co-culture of rat hepatocytes with 3T3 cells by using the microstencil method. The micropatterned configuration of hepatocyte islands was maintained for several weeks in culture by supporting the heterotypic interface. The albumin secretion activities of hepatocytes in the micropatterned co-culture were maintained at higher levels than those observed in random co-culture. Furthermore, the earlier formation of co-culture expressed higher functional activities of hepatocytes than the later formation, and better induction of hepatocyte functions depended on increasing 3T3 cell density of inoculation. These results suggest that the formation of a heterotypic interaction between the hepatocytes and 3T3 cells at an early stage is important to maintain high levels of hepatocyte functions. The findings of this study may provide useful information for designing liver tissue engineering as well as pharmacological and toxicological studies.

\section{Acknowledgments}

This work was partly supported by the Regional Innovation Cluster Program (Global Type-2nd Stage) and by the Nanotechnology Network Project implemented by the Ministry of Education, Culture, Sports, Science, and Technology (MEXT).

\section{References}

1. Enat R, Jefferson DM, Opazo NR, Gatmaitan Z, Leinward LA, et al. (1984) Hepatocyte proliferation in vitro: Its dependence on the use of serum-free hormonally defined medium and substrata of extracellular matrix. Proc Natl Acad Sci 81: 1411-1415.

2. Hamilton GA, Westmorel C, George AE (2001) Effects of medium composition on the morphology and function of rat hepatocytes cultured as spheroids and monolayers. In Vitro Cell Dev Biol Anim 37: 656-667.

3. Mizumoto H, Ishihara K, Nakazawa K, ljima H, Funatsu K, et al. (2008) A new culture technique for hepatocyte organoid formation and long-term maintenance of liver-specific functions. Tissue Eng Part C 14: 167-175.

4. Bissell DM, Stamatoglou SC, Nermut MV, Hughes RC (1986) Interactions of rat hepatocytes with type IV collagen, fibronectin and laminin. Distinct matrixcontrolled modes of attachment and spreading. Eur J Cell Bio 40: 72-78.

5. Bissell DM, Arenson DM, Maher JJ, Roll FJ (1987) Support of cultured hepatocytes by a laminin-rich gel. J Clin Invest 79: 801-812.

6. Sawada N, Tomomura A, Sattler CA, Sattler GL, Kleinman HK, Pitot HC (1987) Effects of extracellular matrix components on the growth and differentiation of cultured rat hepatocytes. In Vitro Cell Dev Biol 23: 267-273.

7. Koide N, Sakaguchi K, Koide Y, Asano K, Kawaguchi M, et al. (1990) Formation of multicellular spheroids composed of adult rat hepatocytes in dishes with positively charged surfaces and under other nonadherent environments. Exp Cell Res 186: 227-235.

8. Tong JZ, Lagausie PD, Furlan V, Cresteil T, Bernard O, et al. (1992) Long-term culture of adult rat hepatocyte spheroids. Exp Cell Res 200: 326-332. 
Citation: Nakazawa K, Shinmura Y, Higuchi A, Sakai Y (2011) Effects of Culture Conditions on a Micropatterned Co-culture of Rat Hepatocytes with 3T3 cells. J Bioprocess Biotechniq S3:002 doi:10.4172/2155-9821.S3-002

Page 5 of 5

9. Sakai Y, Yamagami S, Nakazawa K (2010) Comparative analysis of gene expression in rat liver tissue and monolayer- and spheroid-cultured hepatocytes. Cells Tissues Organs 191: 281-288.

10. Clement B, Guguen-Guillouzo C, Campion JP, Glaise D, et al. (1984) Longterm co-cultures of adult human hepatocytes with rat liver epithelial cells: modulation of albumin secretion and accumulation of extracellular material. Hepatology 4: 373-380.

11. Shimaoka S, Nakamura T, Ichihara A (1987) Stimulation of growth of primary cultured adult rat hepatocytes without growth factors by coculture with nonparenchymal liver cells. Exp Cell Res 172: 228-242.

12. Koike M, Matsushita M, Taguchi K, Uchino J (1996) Function of culturing monolayer hepatocytes by collagen gel coating and coculture with nonparenchymal cells. Artif Organs 20: 186-192.

13. Bhandari RN, Riccalton LA, Lewis AL, Fry JR, Hammond AH, et al. (2001) Liver tissue engineering: a role for co-culture systems in modifying hepatocyte function and viability. Tissue Eng 7: 345-357.

14. Lu HF, Chua KN, Zhang PC, Lim WS, Ramakrishna S, et al. (2005) Threedimensional co-culture of rat hepatocyte spheroids and NIH/3T3 fibroblasts enhances hepatocyte functional maintenance. Acta Biomater 1: 399-410.

15. Thomas RJ, Bhandari R, Barrett DA, Bennett AJ, Fry JR, et al. (2005) The effect of three-dimensional co-culture of hepatocytes and hepatic stellate cells on key hepatocyte functions in vitro. Cells Tissues Organs 181: 67-79.

16. Revzin A, Rajagopalan P, Tilles AW, Berthiaume F, Yarmush ML, Toner M (2004) Designing a hepatocellular microenvironment with protein microarraying and poly(ethylene glycol) photolithography. Langmuir 20: 2999-3005.

17. Otsuka H, Hirano A, Nagasaki Y, Okano T, Horiike Y, et al. (2004) Twodimensional multiarray formation of hepatocyte spheroids on a microfabricated PEG-brush surface. Chembiochem 5: 850-855.

18. Kikuchi K, Sumaru K, Edahiro J, Ooshima Y, Sugiura S, et al. (2009) Stepwise assembly of micropatterned co-cultures using photoresponsive culture surfaces and its application to hepatic tissue arrays. Biotechnol Bioeng 103: 552-561.

19. Bhatia SN, Balis UJ, Yarmush ML, Toner M (1998) Microfabrication of hepatocyte/fibroblast co-culture: role of homotypic cell interactions. Biotechno Prog 14: 378-387.

20. Bhatia SN, Balis UJ, Yarmush ML, Toner M (1998) Probing heterotypic cell interactions: hepatocyte function in microfabricated co-cultures. J Biomater Sci Polym Ed 9: 1137-1160.

21. Khetani SR, Szulgit G, Del Rio JA, Barlow C, Bhatia SN (2004) Exploring interactions between rat hepatocytes and nonparenchymal cells using gene expression profiling. Hepatology 40: 545-554.

22. Takahashi S, Yamazoe H, Sassa F, Suzuki H, Fukuda J (2009) Preparation of coculture system with three extracellular matrices using capillary force lithography and layer-by-layer deposition. J Biosci Bioeng 108: 544-550.

23. Zinchenko YS, Coger RN (2005) Engineering micropatterned surfaces for the coculture of hepatocytes and Kupffer cells. J Biomed Mater Res A 75: 242-248.

24. Cho CH, Berthiaume F, Tilles AW, Yarmush ML (2008) A new technique fo primary hepatocyte expansion in vitro. Biotechnol Bioeng 101: 345-356.

25. Cho CH, Park J, Tilles AW, Berthiaume F, Toner M, Yarmush ML (2010) Layered patterning of hepatocytes in co-culture systems using microfabricated stencils. Biotechniques 48: 47-52.

26. Nakazawa K, Shinmura Y, Yoshiura Y, Sakai Y (2010) Effect of cell spot sizes on micropatterned cultures of rat hepatocytes. Biochem Eng J 53: 85-91.

27. Seglen PO (1976) Preparation of isolated rat liver cells. Methods Cell Biol 13 29-83.

28. Hui EE, Bhatia SN (2007) Micromechanical control of cell-cell interactions. Proc Natl Acad Sci 104: 5722-5726. 